\title{
Proteolytic Regulation of Competence Development in the Genus Streptococcus. Implications in Competence, Stress Response and Antibacterial Therapy
}

\author{
Li $\mathrm{YH}^{1,2^{*}}$ and Tian $\mathrm{XL}^{1}$ \\ ${ }^{1}$ Department of Applied Oral Sciences, Dalhousie University, Canada \\ ${ }^{2}$ Department of Microbiology and Immunology, Dalhousie University, Canada
}

"Corresponding author: Yung-Hua Li, Department of Applied Oral Sciences, Dalhousie University, 5981 University Ave. Rm5215, Halifax, Nova Scotia, Canada, Tel: +1-902-494-3063; E-mail: yung-hua.li@dal.ca

Received date: July 21, 2017; Accepted date: August 16, 2017; Published date: August 17, 2017

Copyright: ( $2017 \mathrm{Li} \mathrm{YH}$, et al. This is an open-access article distributed under the terms of the Creative Commons Attribution License, which permits unrestricted use, distribution, and reproduction in any medium, provided the original author and source are credited.

\begin{abstract}
Competence development for natural genetic transformation is widespread in bacteria, although it is a transient physiological state in which bacteria are capable of taking up and integrating exogenous DNA. Activation of competence enables the recipient bacteria to gain new genes, promoting the emergence of genetic variation, antibiotic resistance and evolution of virulence factors. It has been well studied that competence activation in bacteria requires one or more signal transduction pathways, many competence proteins and a complex regulatory network, which are finely controlled by a competence-specific master regulator. In the Genus Streptococcus, an alternative sigma factor, SigX coded by $\operatorname{com} X$, is such a master regulator. The transcriptional activation of com $X$ is the key step of competence induction in nearly all members of Streptococci. Although much is known of the regulatory cascade leading to competence induction, it was not until recently that a negative regulation of competence by a mechanism that involves regulated proteolysis of SigX has been recognized to play an important role in competence development in these bacteria. In particular, an adaptor protein MecA is found to target SigX for degradation by the proteases $\mathrm{ClpC/ClpP,} \mathrm{profoundly} \mathrm{affecting} \mathrm{the} \mathrm{stability} \mathrm{of} \mathrm{SigX} \mathrm{during} \mathrm{competence} \mathrm{development.}$ Interestingly, SigX-mediated competence appears to be antagonistically connected with antibiotic-induced stress response by an unknown mechanism that involves activities of MecA and the Clp protease family. This discovery has added a new level of the complexity of competence regulatory network in Streptococci, leading to further investigation of such proteolytic regulation in competence, stress response, and antibacterial therapy.
\end{abstract}

Keywords: Competence; Regulated proteolysis; Adaptor protein; Proteases; Streptococci

\section{Introduction}

Competence development for natural genetic transformation is a powerful process by which bacteria are capable of taking up, transporting and integrating exogenous DNA [1,2]. Analysis of microbial genomes reveals that horizontal gene transfer through genetic transformation is widespread in bacteria. This process enables the recipient bacteria to gain new genes, promoting the emergence of genetic variation, antibiotic resistance, and evolution of virulence factors [1-4]. Therefore, natural transformation is an important mechanism in which bacteria adapt to changing environments. Since the first discovery of genetic transformation in Streptococcus pneumoniae in 1928, the cellular processes and molecular regulatory mechanisms involved in genetic transformation have been extensively studied in several model organisms $[1,2]$.

It has been well studied that competence development in the Genus Streptococcus is activated through at least two different quorumsensing signal transduction pathways, the ComCDE and the ComRS $[5,6]$. The ComCDE is the well-studied quorum sensing system in the mitis group of Streptococci, such as Streptococcus pneumoniae and Streptococcus mitis, whereas the ComRS is the recently discovered quorum sensing system that functions similarly in the salivarius, mutans, pyogenic and bovis groups of Streptococci [6]. Both quorum- sensing systems are demonstrated to induce the competence cascades by activating comX that encodes a competence-specific master regulator $\operatorname{SigX}(\sigma \mathrm{X})$ [5,7]. For the species that primarily use the ComCDE pathway, the cells during exponential growth can secrete a competence-stimulating peptide or CSP that is detected by the ComD histidine kinase receptor of the neighboring cells $[3,7,8]$. This leads to autophosphorylation of ComE response regulator that in turn activates transcription of comX, triggering the competence cascade [3]. For the species that mainly use the ComRS pathway, the cells growing in chemically defined medium (CDM) can sense a comX-inducing peptide or XIP inside the cells after XIP is internalized by an oligopeptide permease transporter Opp. The XIP then interacts with ComR to form a ComR/XIP complex that activates transcription of comX, triggering the competence cascade $[5,6]$. Clearly, all groups of Streptococci require transcriptional activation of comX to induce competence for genetic transformation, despite different signaling pathways involved during competence development [4-8]. Therefore, SigX is the competence-specific master regulator that is absolutely required for driving transcription of many late competence genes necessary for DNA transport, update and recombination [9].

Interestingly, Streptococcus mutans, a leading cariogenic pathogen that can cause dental caries worldwide, has evolved to have both the ComCDE and ComRS signaling systems that are confirmed to induce competence in this bacterium (Figure 1). When grown in a complex medium, Streptococcus mutans cells secrete detect and respond to CSP through the Com $\mathrm{AB}$ transporter and ComDE signal transduction 
pathway [8]. CSP can be detected by the ComD receptor of a twocomponent system ComDE, leading to autophosphorylation of its response regulator ComE, which in turn activates transcription of genes encoding an array of nonlantibiotic bacteriocins [10,11]. The activated ComE also strongly induces expression of ComX, resulting in competence activation of a subpopulation [11-13].

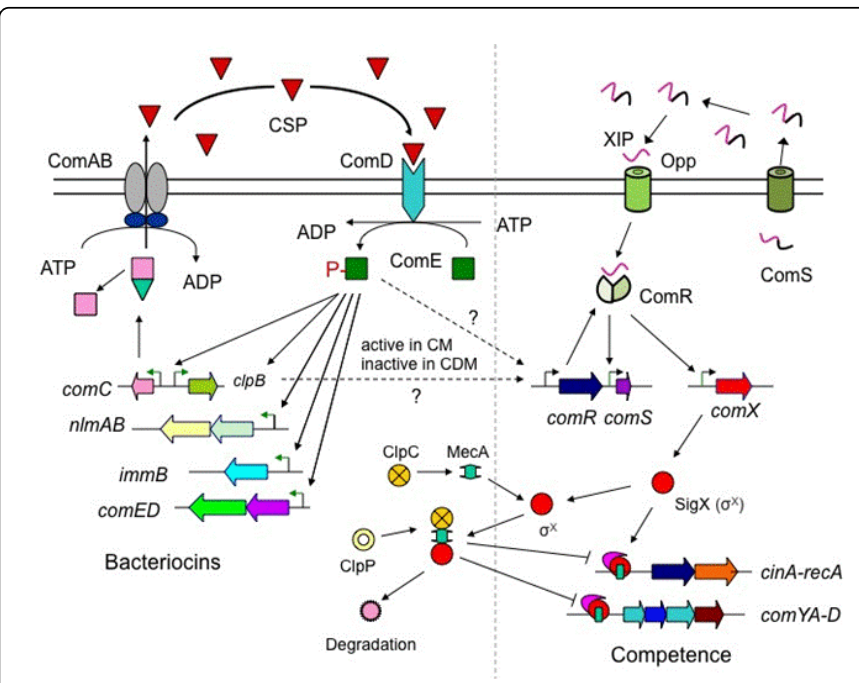

Figure 1: A schematic diagram describes the proposed competence regulatory network in Streptococcus mutans, which can use both the ComCDE and ComRS signaling systems to regulate competence development. These two signaling transduction pathways along with their dedicated signaling peptides, CSP and XIP, are described. The key step for competence induction is the transcriptional activation of comX, which encodes a competence specific master regulator, SigX. In a complex medium (CM), CSP interacts with the ComDE that activate the transcription of comX by an unknown mechanism, triggering the competence cascade in less than $10 \%$ of the population. In a chemical defined medium (CDM), the ComRS system can sense and respond XIP inside the cells. Once internalized via an oligopeptide permease transporter Opp, the XIP interacts with ComR to form a ComR/XIP complex. This complex then binds to the ComR-box and drives the transcription of comX and comS, triggering the competence cascade in nearly the entire population. In a complex medium (CM), however, a low level of SigX can be sequestrated in a ternary complex SigX-MecA-ClpC, which in turn triggers degradation of SigX in the presence of ClpP, thereby, negatively regulating competence development Adapted from Tian et al. [14].

However, the pathway transmitting this signal from the ComE to comX was unclear, since there is no the ComE binding site in the promoter region of comX in Streptococcus mutans [15]. This missing link has been recently filled by the discovery of a type II ComRS signaling system that proximally induces transcription of comX in Streptococcus mutans in response to signal peptide XIP [5,6]. When Streptococcus mutans is grown in CDM, competence activation through the ComRS system is superior, nearly involving the entire population $[5,16]$. However, XIP appears to be inactive when Streptococcus mutans is grown in a complex medium [5,16-18]. This phenomenon is interpreted as a bimodal behavior of Streptococcus mutans in response to different signaling peptides due to changes in medium components [18]. Importantly, such bimodal behavior requires the presence of intact comS that encodes the precursor of XIP. Thus, it becomes clear that Streptococcus mutans can finely regulate genetic competence via a complex regulatory network that receives and responds inputs from at least two signaling peptides, CSP and XIP, dependently on growth conditions. The key step of competence induction by either of these signaling peptides is transcriptional activation of comX that controls expression of late competence genes for DNA transport, uptake and recombination [16-18].

Considerable studies reveal that besides transcriptional activation of comX, SigX regulator protein is also the target of posttranslational regulation in Streptococcus pneumoniae, which develops competence by receiving the signal from the ComCDE transduction pathway [9]. In this case, SigX is positively controlled by ComW but negatively regulated by the protease ClpE/ClpP $[19,20]$. The stability of SigX in Streptococcus pneumoniae can be also negatively affected by ComW, since ComW is labile to degradation by the ClpC/ClpP complex [21]. Post-translational regulation of competence is well studied in Bacillus subtilis, in which a master regulator ComK is required for competence induction [22-24]. Under non-competence conditions, ComK is sequestered in a MecA and $\mathrm{ClpC}$ complex, which triggers degradation of ComK in the presence of ClpP. The ComK can activate competence only when it is released from this complex by action of a small protein ComS, which binds to adaptor protein MecA, releasing the regulator ComK [24].

However, it was unclear whether the same mechanism might be involved in regulation of the stability and activity of SigX in the streptococcal species that primarily use a ComRS system to control transcriptional activation of comX. Two independent research groups have investigated this question in two species of Streptococci, Streptococcus thermophilus and Streptococcus mutans. Both groups of researchers have discovered that an adaptor protein $\mathrm{MecA}$ is required to regulate genetic competence by a mechanism that requires the presence of a functional SigX in these species $[24,25]$. They have found that changes in expression of MecA dramatically affect the stability and activity of SigX. These findings led to the hypothesis that MecA might target SigX for degradation by the $\mathrm{ClpC} / \mathrm{ClpP}$ proteases in these members of Streptococci.

To explore the molecular detail of such negative regulation of competence in these bacteria, Dong et al. have further investigated MecA-mediated proteolysis of SigX and the impact on competence regulation in Streptococcus mutans [26]. They first analyze the cellular levels of SigX. Their work shows that the synthesis of SigX is transiently induced by CSP, but SigX is rapidly degraded during the exponential growth in a complex medium, a sub-permissive condition for competence activation [24-26]. A deletion of mecA, clpC or clpP results in the cellular accumulation of SigX and a prolonged competence state, while an overexpression of MecA enhances the proteolysis of SigX and accelerates the escape from competence. However, a deletion of mecA alone is insufficient to activate competence under this growth condition. These researchers have provided strong evidence that Streptococcus mutans regulates competence by a mechanism that involves proteolysis of SigX when grown in a complex medium, in which the synthesis of SigX is transiently induced by CSP but it is quickly degraded by MecAmediated proteolysis in the presence of $\mathrm{ClpC}$ and $\mathrm{ClpP}$. Their work confirms that MecA can interact with both SigX and ClpC, enabling these proteins to form a ternary complex of SigX-MecA-ClpC [26]. This ternary complex in turn sequesters the activity of SigX and triggers ATP-dependent degradation of SigX in the presence of ClpP. 
Citation: Li YH, Tian XL (2017) Proteolytic Regulation of Competence Development in the Genus Streptococcus: Implications in Competence, Stress Response and Antibacterial Therapy. J Antimicrob Agents 3: 148. doi:10.4172/2472-1212.1000148

Page 3 of 5

This work further confirms that MecA binds to SigX protein via its $\mathrm{N}$ terminal domain (NTD1-82) and to ClpC protein via its C-terminal domain (MecA-CTD123-240). A deletion of either the NTD or CTD abolishes the binding of MecA to SigX or ClpC, failing to mediate the formation of such a ternary complex. These findings suggest that the NTD of MecA is essential for substrate recognition, while the CTD is required for the assembly and activity of the ClpC/ClpP complex [26]. A sequence alignment of MecA proteins confirms that MecA proteins are well conserved among four Streptococcus mutans strains examined (Figure 2). In particular, MecA proteins share high levels of conservation at the NTD and CTD with those of Streptococcus thermophilus and Bacillus subtilis [23,25,27], suggesting that MecA in Streptococcus mutans truly acts as an adaptor to interact with its partner proteins for regulated proteolysis of SigX in a manner similarly to those in Streptococcus thermophilus and Bacillus subtilis [23-25]. These findings support the notion that MecA plays central role in regulated proteolysis of SigX by its substrate recognition and the Clp protease complex assembly and activity. Thus, MecA actually acts as an anti-sigma factor to regulate the stability of $\operatorname{SigX}$ during competence development in these bacteria.

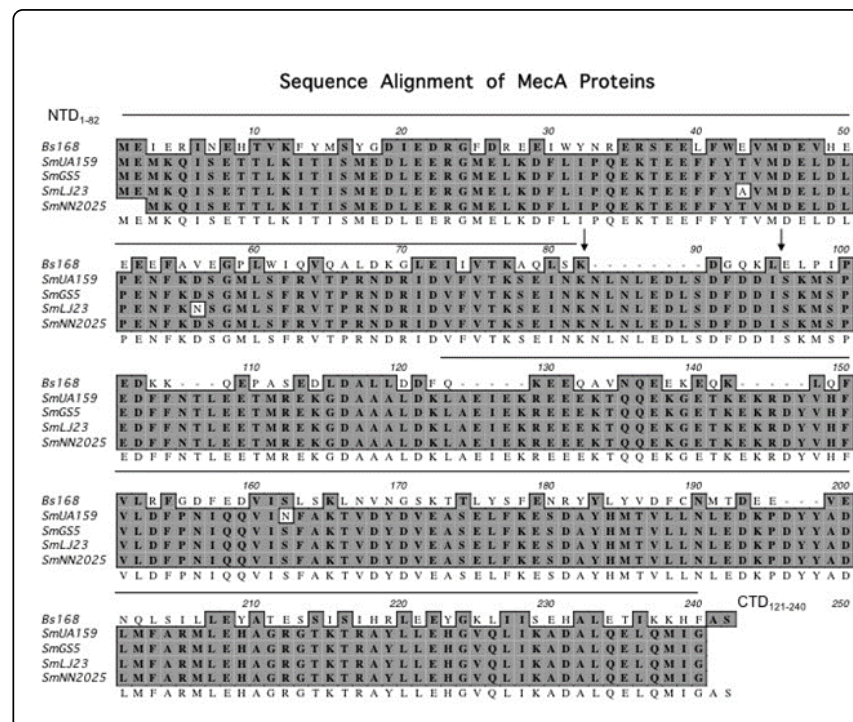

Figure 2: A sequence alignment of MecA proteins from four Streptococcus mutans strains, including UA159, GS-5, LJ23 and NN2025, was performed against that of Bacillus subtilis 168 (Bs168) using MacVector 9.0 ClusterW. The conservations of amino acid residues cross all the strains are highlighted in the grey box. Black lines above the sequence alignment indicate the $\mathrm{N}$-terminal domain (NTD1-82) and the C-terminal domain (CTD123-240) of MecA proteins. Small vertical arrows indicate the preferred cleavage sites of Bacillus subtilis MecA protein by trypsin (K82) and V8 protease (E93). The sequences of all MecA proteins are from gene bank accession numbers of Bacillus subtilis 168 (NP_389034.1), Streptococcus mutans UA159 (NP_72079.1), GS-5 (YP_006489516.1), LJ23 (YP_006252000.1) and NN2025 (YP_003485630.1).

The implications of an adaptor protein that acts as an anti-sigma factor in a Clp protease-dependent manner were previously reported in both Gram-negative and Gram-positive bacteria [27-29]. In Gramnegative bacteria such as Escherichia coli, for example, adaptor protein
RssB can specifically target Sigma $S$ or RpoS for degradation by the proteinase ClpX/ClpP, regulating the levels of RpoS in the stationary phase cells or in general stress response of Escherichia coli [29]. Another example is adaptor protein RsiW that acts as an anti-sigma factor in the control of sigma factor $\mathrm{W}(\sigma \mathrm{W})$ of Bacillus subtilis during $\mathrm{pH}$ and salt stresses [30]. Similarly, Bacillus subtilis controls the activity of an alternative sigma factor, $\sigma \mathrm{V}$ to cope with antibioticinduced envelope stress by regulating proteolysis of sigma factor $\sigma \mathrm{V}$ through action of an anti-sigma factor RsiV or an adaptor protein $[30,31]$. Thus, regulated proteolysis of a master regulator by an adaptor protein that acts as an anti-sigma factor in regulation of physiology and stress response is well conserved among all bacteria, including the members of the Genus Streptococcus [32]. These studies reinforce the importance of fine control of regulated proteolysis in bacterial physiology, stress response and virulence evolution. However, the role of MecA in regulation of stress response other than competence in the Genus Streptococcus remains to be studied. Regulated proteolysis by ATP-dependent proteases is widely distributed in both prokaryotic and eukaryotic cells, and plays important roles in the timely turnover of many intracellular proteins $[33,34]$. Good examples of such proteases in bacteria include the Clp superfamily proteases, such as ClpAP, $\mathrm{ClpCP}, \mathrm{ClpXP}, \mathrm{ClpB}, \mathrm{ClpE}$ and $\mathrm{ClpL}$, which are found to regulate bacterial growth, sporulation, competence, virulence factors and stress response [33-35]. These proteases ensure the survival and physiology of a cell by removing misfolded or regulatory proteins, whose degradation is vital to the control of signaling cascades, metabolism, cellular development or cell fate under a specific condition [33]. For degradation of correct proteins at correct times, eukaryotic cells use the ubiquitin-tagging machinery to recognize and target proteins for degradation by the proteasome [29]. However, most bacteria carry out selective degradation of proteins by using an adaptor that recognizes specific substrates for degradation by proteases [29-32]. Several adaptor proteins have been identified in many bacteria, including SspB and ClpS in Escherichia coli, and MecA, YjbH, YpbH and McsB in Bacillus subtilis [36,37]. MecA in Bacillus subtilis is a general adaptor protein to mediate the Clp protease-dependent proteolysis, allowing degradation of unfolded or specific proteins, such as competencespecific regulator ComK $[28,32,37]$. This system has parallels to the regulation of RpoS degradation that depends on an adaptor protein in Escherichia coli $[29,34]$. Clearly, these adaptor proteins provide a way to modulate substrate specificity for regulated proteolysis and play important roles in quality control of cellular proteins or the activities. Therefore, control of the availability and activity of adaptor proteins may be crucial for bacteria to respond to changing environments or stresses.

Recent studies show that certain cell wall-acting antibiotics, such as bacitracin, at a sub-MIC concentration up-regulate expression of $\mathrm{MecA}$, but suppress CSP-induced competence in Streptococcus mutans. Initial evidence suggests that bacitracin a sub-MIC does not only suppress competence, but also induces stress response by a mechanism that requires the presence of MecA. It has been known that bacitracin at a sub-MIC often induces cell envelope stress response by several mechanisms $[38,39]$.

However, there is no report whether bacitracin affects genetic competence, although several DNA-damaging antibiotics, such as mitomycin $\mathrm{C}$ and fluoroquinolones, are known to induce genetic transformation in Streptococcus pneumoniae and other bacteria [40, 41]. Competence induction by these DNA damaging antibiotics is considered as stress response in parallel to the SOS response in Bacillus subtilis and Escherichia coli. In contrast to these reports, Boutry et al. 
Citation: Li YH, Tian XL (2017) Proteolytic Regulation of Competence Development in the Genus Streptococcus: Implications in Competence, Stress Response and Antibacterial Therapy. J Antimicrob Agents 3: 148. doi:10.4172/2472-1212.1000148

Page 4 of 5

have found that SOS response activation and competence induced by DNA-damaging agents are an antagonistic mechanism in Streptococcus thermophilus, which uses type I ComRS system to activate transcription of comX [42]. However, it remains an open question whether bacitracin suppresses competence by up-regulating expression of MecA and its regulated proteolysis in these bacteria. Further study is required to determine how antibiotics affect MecA, $\mathrm{ClpC}$ and other Clp family proteases in these bacteria.

\section{Conclusion}

In summary, many members of the Genus Streptococcus are naturally transformable, dependently on competence induction involving a regulatory mechanism requiring a master regulator SigX. Recent studies show that adaptor protein MecA in these species plays a crucial role in recognizing and targeting SigX master regulator for degradation by a protease complex. Thus, MecA actually acts as an anti-sigma factor to regulate the stability and activity of SigX in competence development in these bacteria. Therefore, changes in expression of MecA may profoundly affect the stability and activity of SigX. However, it remains to be determined what affect expression of $\mathrm{MecA}$ and whether stress response, competence and regulated proteolysis are correlated in the same regulatory circuit in these bacteria. We speculate that resolving these questions and related molecular mechanisms may improve our understanding of bacterial stress response, competence and regulated proteolysis, offering an opportunity to identify new targets, such as adaptor proteins or proteolytic proteases, for antibacterial therapies.

\section{Acknowledgments}

This work was supported by the Canadian Institute for Health Research (CIHR) operating grant MOP-115007 and by the Natural Sciences and Engineering Research Council of Canada (NSERC) discovery grant RGPIN 311682-07. The authors declare that they have no competing financial interest.

\section{References}

1. Chen I, Dubnau D (2004) DNA uptake during bacterial transformation. Nat Rev Microbiol 2: 241-249.

2. Johnsborg O, Eldholm, V, Havarstein, LS (2007) Natural genetic transformation: Prevalence, mechanisms and function. Res Microbiol 158: 767-778.

3. Martin B, Quentin Y, Fichant G, Claverys JP (2006) Independent evolution of competence regulatory cascades in Streptococci? Trend Microbiol 14: 339-345.

4. Claverys JP, Prudhomme M, Martin B. (2006) Induction of competence regulons as a general response to stress in Gram-positive bacteria. Annu Rev Microbiol 60: 451-475.

5. Mashburn-Warren L, Morrison DA, Federle MJ (2010) A novel doubletryptophan peptide pheromone controls competence in Streptococcus spp. via an Rgg regulator. Mol Microbiol 78: 589-606.

6. Federie MJ, Marrison DA (2012) One if by land, two if by sea: Signaling to the ranks with CSP and XIP. Mol Microbiol 86: 241-245.

7. Lee MS, DA Morrison (1999) Identification of a new regulator in Streptococcus pneumoniae linking quorum sensing to competence for genetic transformation. J Bacteriol 181: 5004-5016.

8. Li YH, Lau PCY, Lee JH, Ellen RP, Cvitkovitch DG (2001) Natural genetic transformation of Streptococcus mutans growing in biofilms. J Bacteriol 183: 897-908.

9. Luo P, Morrison DA (2003) Transient association of an alternative sigma factor, ComX, with RNA polymerase during the period of competence for genetic transformation in Streptococcus pneumoniae. J Bacteriol 185: 349-358.

10. Tian XL, Syvitski RT, Liu T, Livingstone N, Jakeman DL, et al. (2009) A method for structure-activity analysis of quorum-sensing signaling peptides from naturally transformable Streptococci. Biol Procedure Online 11: 207-226.

11. Ploeg JR (2005) Regulation of bacteriocin production in Streptococcus mutans by the quorum-sensing system required for development of genetic competence. J Bacteriol 187: 3980-3989.

12. Syvitski RT, Tian XL, Sampara K, Salman A, Lee SF, et al. (2007) Structure-activity analysis of quorum-sensing signaling peptides from Streptococcus mutans. J Bacteriol 189: 1441-1450.

13. Lemme A, Grobe L, Reck M, Tomasch J, Dobler IW (2011) Subpopulation-specific transcriptome analysis of competencestimulating-peptide-induced Streptococcus mutans. J Bacteriol 193: 1863-1877.

14. Tian XT, Dong GF, Liu TL, Gomez ZA, Wahl A, et al. (2013) MecA protein acts as a negative regulator of genetic competence in Streptococcus mutans. J Bacteriol 195: 5196-5206.

15. Hung DCI, Downey JS, Ayala EA, Kreth J, Mair R, et al. (2011) Characterization of DNA binding sites of the ComE response regulator from Streptococcus mutans. J Bacteriol 193: 3642-3652.

16. Desai K, Warren LM, Federle MJ, Morrison DA (2012) Development of competence for genetic transformation of Streptococcus mutans in a chemically defined medium. J Bacteriol 194: 3774-3780.

17. Khan R, Rukke HV, Filho APR, Fimland G, Arntzen MO, et al. (2012) Extracellular identification of a processed type II ComR/ComS pheromone of Streptococcus mutans. J Bacteriol 194: 3781-3788.

18. Son M, Ahn SJ, Guo Q, Burne RA, Hagen SJ (2012) Microfluidic study of competence regulation in Streptococcus mutans: environmental inputs modulate bimodal and unimodal expression of comX. Mol Microbiol 86: 258-272.

19. Luo P, Li H, Morrison DA (2004) Identification of ComW as a new component in the regulation of genetic transformation in Streptococcus pneumoniae. Mol Microbiol 54: 172-183.

20. Piotrowski A, Luo P, Morrison DA (2009) Competence for genetic transformation in Streptococcus pneumoniae: Termination of activity of the alternative sigma factor ComX is independent of proteolysis of ComX and ComW. J Bacteriol 191: 3359- 3366.

21. Chastanet A, Prudhomme M, Claverys JP, Msadek T (2001) Regulation of Streptococcus pneumoniae clp genes and their role in competence development and stress survival. J Bacteriol 183: 7295-7307.

22. Turgay K, Hahn J, Burghoorn J, Dubnau D (1998) Competence in Bacillus subtilis is controlled by regulated proteolysis of a transcriptional factor. The EMBO J 17: 6730-6738.

23. Liu J, Zuber P (1998) A molecular switch controlling competence and motility: Competence regulatory factors ComS, MecA, and ComK control sigma D-dependent gene expression in Bacillus subtilis. J Bacteriol 180: 4243-4251.

24. Turgay K, Persuh M, Hahn J, Dubnau D (2001) Roles of the two ClpC ATP binding sites in the regulation of competence and the stress response. Mol Microbiol 42: 717-727.

25. Boutry C, Wahl A, Delplace B, Clippe A, Fontaine L, et al. (2011) Adaptor protein $\mathrm{MecA}$ is a negative regulator of the expression of late competence genes in Streptococcus thermophilus. J Bacteriol 193:1777-1788.

26. Dong GF, Tian XL, Gomez ZA, Li YH (2014) Regulated proteolysis of the alternative sigma factor SigX in Streptococcus mutans: implication in the escape from competence. BMC Microbiol 14: 183.

27. Biornstad TJ, Havarstein LS (2011) ClpC acts as a negative regulator of competence in Streptococcus thermophilus. Microbiol 157: 1676-1684.

28. Turgay K, Hamoen LW, Venema G, Dubnau D (1997) Biochemical characterization of a molecular switch involving the heat shock protein $\mathrm{ClpC}$, which controls the activity of ComK, the competence transcription factor of Bacillus subtilis. Genes Develop 11: 119-128. 
Citation: Li YH, Tian XL (2017) Proteolytic Regulation of Competence Development in the Genus Streptococcus: Implications in Competence, Stress Response and Antibacterial Therapy. J Antimicrob Agents 3: 148. doi:10.4172/2472-1212.1000148

Page 5 of 5

29. Battesti A, Gottesman S (2013) Roles of adaptor proteins in regulation of bacterial proteolysis. Curr Opinin Microbiol 16: 140-147.

30. Ades SE (2008) Regulation by destruction: Design of the $\sigma \mathrm{E}$ envelope stress response. Curr Opin Microbiol 11: 535-540.

31. Hastie JL, Williams KB, Ellermeier CD (2013) The activity of $\sigma \mathrm{V}$, an extracytoplasmic function $\sigma$ factor of Bacillus subtilis, is controlled by regulated proteolysis of the anti- $\sigma$ factor RsiV. J Bacteriol 195: 3135-3144.

32. Schlothauer T, Mogk A, Dougan DA, Bukau B, Turgay K (2003) MecA, an adaptor protein necessary for ClpC chaperone activity. Proc Natl Acad Sci USA 100: 2306-2311.

33. Ehrmann M, Clausen T (2004) Proteolysis as a regulatory mechanism. Annu Rev Genet 38: 709-724.

34. Zhou YN, Gottesman S (1998) Regulation of proteolysis of the stationaryphase sigma factor RpoS. J Bacteriol 180: 1154-1158.

35. Frees D, Savijoki K, Varmanen P, Ingmer H (2007) Clp ATPases and ClpP proteolytic complexes regulate vital biological processes in low GC, Gram-positive bacteria. Mol Microbiol 63: 1285-1295.

36. Cezairliyan BO, Sauer RT (2007) Inhibition of regulated proteolysis by RseB. Proc Natl Acad Sci USA. 104: 3771-3776.
37. Kirstein J, Moliere N, Dougan DA, Turgay K (2009) Adapting the machine: adaptor proteins for Hsp100/Clp and AAA+ proteases. Nat Rev Microbiol 7: 589-599.

38. Jordan S, Hutchings MI, TMascher T (2008) Cell envelope stress response in Gram- Positive bacteria. FEMS Microbiol Rev 32: 107-146.

39. Ouyang J, Tian XL, Versey J, Wishart A, Li YH (2010) The BceABRS fourcomponent system regulates bacitracin-induced cell envelope stress response in Streptococcus mutans. Antimicrob Agents Chemother 54: 3895-3906.

40. Prudhomme M, Attaiech L, Sanchez G, Martin B, Claverys JP (2006) Antibiotic stress induces genetic transformability in the human pathogen Streptococcus pneumoniae. Science 313: 89-92.

41. Charpentier X, Polard P, Claverys JP (2012) Induction of competence for genetic transformation by antibiotics: convergent evolution of stress responses in distant bacterial species lacking SOS? Curr Opin Microbiol 15: $570-576$

42. Boutry C, Delplace B, Clipe A, Fontaine L, Hols P (2013) SOS response activation and competence development are antagonistic mechanisms in Streptococcusthermophilus. J Bacteriol 195: 696-707. 